\title{
Sur une conjecture de Pohst
}

\author{
par
}

\section{J. Bertin (Paris)}

Introduction. Soit $n$ nombres réels $x_{1}, \ldots, x_{n}$ distincts, ordonnés par ordre croissant

$$
\left|x_{1}\right|<\ldots<\left|x_{n}\right| .
$$

Considérons la quantité

$$
\begin{aligned}
D_{n} & =\prod_{1 \leq i<j \leq n}\left(x_{i}-x_{j}\right)^{2}=\left(\prod_{j=2}^{n}\left|x_{j}\right|^{2(j-1)}\right) \prod_{1 \leq i<j \leq n}\left(1-x_{i} / x_{j}\right)^{2} \\
& =\left(\prod_{j=2}^{n}\left|x_{j}\right|^{2(j-1)}\right) P_{n} .
\end{aligned}
$$

Pohst [3] a montré, pour $n \leq 11$, l'inégalité

$$
P_{n} \leq 4^{[n / 2]}
$$

et conjecturé que l'inégalité précédente était valable pour tout $n \geq 2$.

Une telle inégalité intervient à propos d'estimations de régulateurs de corps de nombres. Mais elle peut être utile également pour des majorations de discriminants de corps de nombres totalement réels, grâce à la détermination de nombres de Pisot [2] $\theta=\theta_{1}$ totalement réels, de degré $n$, de "mesure pondérée" $\mu(\theta)$ minimale, où $\mu(\theta)=\theta_{1}^{n-1} \theta_{2}^{n-2} \ldots \theta_{n-1}$ si $\theta_{2}, \ldots, \theta_{n-1}$ désignent les conjugués de $\theta$ vérifiant $\left|\theta_{n-1}\right|<\left|\theta_{n-2}\right|<\ldots<$ $\left|\theta_{2}\right|<1<\theta_{1}=\theta$.

La démonstration de l'inégalité repose sur le lemme suivant et utilise un argument de théorie des simplexes. Le lemme permet en outre de donner une nouvelle démonstration de l'inégalité due à Remak [1] : $\left|P_{n}\right| \leq n^{n}$ lorsque les $x_{i}$ ne sont plus réels mais complexes.

Lemme. Avec les notations précédentes, on a l'égalité

$$
P_{n}=D_{n} /\left(\prod_{j=2}^{n}\left|x_{j}\right|^{2(j-1)}\right)=\left(\operatorname{det}\left(a_{i j}\right)\right)^{2}
$$


où

$$
\begin{gathered}
\varrho_{i}=x_{i} / x_{i+1}, \quad 1 \leq i \leq n-1, \\
a_{i j}=\varrho_{i}^{j-i} \varrho_{i+1}^{j-i-1} \ldots \varrho_{j-1}, \quad j>i, \\
a_{i j}=\varrho_{j+1} \varrho_{j+2}^{2} \ldots \varrho_{i-1}^{i-j-1}, \quad j<i-1, \\
a_{i i}=a_{i, i-1}=1 .
\end{gathered}
$$

Preuve. Posons $\delta_{n}=\left|\operatorname{det}\left(x_{i}^{j-1}\right)\right|=\sqrt{D_{n}}$ et montrons que

$$
\left|\delta_{n} / x_{2} x_{3}^{2} \ldots x_{n}^{n-1}\right|=\left|\operatorname{det}\left(a_{i j}\right)\right| .
$$

Soit $X=\left(x_{i}^{j-1}\right)$. Soit $B$ la matrice diagonale telle que $b_{11}=1$ et $b_{i i}=$ $1 / x_{i} x_{i-1} \ldots x_{2}$ pour $i \geq 2$. Soit $C$ la matrice diagonale telle que $c_{11}=c_{22}=1$ et $c_{i i}=\varrho_{i-1}^{i-2} \varrho_{i-2}^{i-3} \ldots \varrho_{2}, i \geq 3$. Comme $\operatorname{det}(B) \operatorname{det}(C)=1 / x_{2} x_{3}^{2} \ldots x_{n}^{n-1}$, il suffit de montrer que $C X B=A$. Posons $X B=\left(d_{i j}\right)$. On a

$$
\begin{gathered}
d_{i j}=\sum_{k} x_{i k} b_{k j}=x_{i j} b_{j j}=x_{i}^{j-1} / x_{j} x_{j-1} \ldots x_{2}, \quad j \geq 2, \\
d_{i 1}=1 .
\end{gathered}
$$

Posons $C(X B)=\left(r_{i j}\right)$. On vérifie que $r_{i j}=c_{i i} d_{i j}=a_{i j}$.

Corollaire. Soit $n$ nombres complexes $x_{1}, \ldots, x_{n}$ vérifiant $\left|x_{1}\right| \leq\left|x_{2}\right| \leq$ $\ldots \leq\left|x_{n}\right|$. Alors

$$
\left|P_{n}\right|=\prod_{1 \leq i<j \leq n}\left|1-x_{i} / x_{j}\right|^{2} \leq n^{n} .
$$

Preuve. On garde les notations du lemme. Comme $\left|a_{i j}\right| \leq 1$, il suffit d'appliquer l'inégalité de Hadamard.

ThÉORÈme. Avec les notations précédentes, si les $x_{i}$ sont réels, $1 \leq i \leq$ $n$, on a l'inégalité

$$
P_{n} \leq 4^{[n / 2]},
$$

où $[x]$ désigne la partie entière de $x$.

Preuve. Selon les notations du lemme, $\delta_{n}=\left|\operatorname{det}\left(\varrho_{1}, \ldots, \varrho_{n}\right)\right|$.

Comme le déterminant est une combinaison linéaire des éléments d'une ligne, on considère d'abord le simplexe des éléments positifs de la $i$-ième ligne $\left\{a_{i j}: 0 \leq a_{i j} \leq 1, j \in J(i)\right\}$. Comme le déterminant ne peut prendre son maximum qu'en un sommet du simplexe, on en déduit que pour la valeur maximum du déterminant, les éléments positifs de la $i$-ième ligne valent 0 ou 1. Un raisonnement analogue entraîne que pour le maximum de $\delta_{n}$, les éléments négatifs de la $i$-ième ligne valent 0 ou -1 .

D'après l'expression précédente de $\delta_{n}$ trouvée dans le lemme, on en déduit que $\delta_{n}$ est maximum pour $\varrho_{i} \in\{-1,0,1\}, 1 \leq i \leq n$.

Notons alors $\gamma_{n}$ le maximum de $\delta_{n}$.

Si $\varrho_{n-1}=0$, alors $\delta_{n}=\left|\operatorname{det}\left(\varrho_{1}, \ldots, \varrho_{n-1}\right)\right|$; d'où $\delta_{n} \leq \gamma_{n-1}$. 
Si $\varrho_{n-1}=1$, alors l'avant-dernière et la dernière ligne de $\delta_{n}$ sont identiques; par suite $\delta_{n}=0$.

Supposons donc $\varrho_{n-1}=-1$.

(a) Si $\varrho_{n-2}=1$, les $(n-2)$-ième et $(n-1)$-ième lignes de $\delta_{n}$ sont égales; d'où $\delta_{n}=0$.

(b) Si $\varrho_{n-2}=-1$, la $(n-2)$-ième ligne est l'opposée de la dernière ligne; d'où $\delta_{n}=0$.

(c) Si $\varrho_{n-2}=0, \delta_{n}$ s'écrit

$$
\delta_{n}=\left|\begin{array}{cccc} 
& 0 & 0 & 0 \\
\delta_{n-2} & \ldots \ldots & \ldots \\
& 0 & 0 & 0 \\
0 \ldots 0 & 1 & 1 & -1 \\
0 \ldots 0 & -1 & 1 & 1
\end{array}\right|,
$$

d'où après avoir ajouté l'avant-dernière ligne à la dernière ligne, on obtient $\delta_{n}=2 \delta_{n-2} \leq 2 \gamma_{n-2}$.

Par suite $\gamma_{n}=\sup \left(\gamma_{n-1}, 2 \gamma_{n-2}\right)$.

Raisonnons alors par récurrence. On a $\gamma_{2}=\gamma_{3}=2$; supposons $\gamma_{i}=2^{[i / 2]}$ pour $2 \leq i \leq n-1$.

Si $n=2 k$, on en déduit $\gamma_{2 k}=2 \gamma_{2 k-2}=2^{[n / 2]}$.

Si $n=2 k+1$, on a alors $\gamma_{2 k+1}=2 \gamma_{2 k-1}=\gamma_{2 k}=2^{[n / 2]}$.

En résumé, si $n$ est pair, le maximum est atteint pour les $\varrho$ d'indice pair nuls et les $\varrho$ d'indice impair égaux à -1 .

\section{Références}

[1] A.-M. Bergé et J. Martinet, Sur les minorations géomètriques des régulateurs, dans : Séminaire de Théorie des Nombres, Paris 1987-88, Progr. Math. 81, Birkhäuser, 1990, 23-50.

[2] M. J. Bertin, A. Decomps-Guilloux, M. Grandet-Hugot, M. PathiauxDelafosse, J. P. Schreiber, Pisot and Salem Numbers, Birkhäuser, 1992.

[3] M. Pohst, Regulatorabschätzungen für total reelle algebraische Zahlkörper, J. Number Theory 9 (1977), 459-492.

Equipe d'Arithmétique

U.M.R. 9994

Université Paris 6

4 Place Jussieu

75252 Paris Cedex 05, France

E-mail: majb@ccr.jussieu.fr 